\title{
Application- and Network-Cognizant Proxies - Final Report
}

\author{
Antonio Ortega \\ Associate Professor \\ University of Southern California \\ Integrated Media Systems Center/Signal \& Image Processing Institute \\ Department of Electrical Engineering - Systems \\ 3740 McClintock Avenue \\ Los Angeles, CA 90089-2564 \\ Voice: (213) 740-2320 \\ Fax: (213) 740-4651 \\ E-mail: ortega@sipi.usc.edu \\ Daniel C. Lee \\ Assistant Professor \\ University of Southern California \\ Communication Sciences Institute \\ Department of Electrical Engineering - Systems \\ 3740 McClintock Avenue \\ Los Angeles, CA 90089-2565 \\ Voice: (213) 740-0882 \\ Fax: (213) 740-8729 \\ E-mail: dclee@usc.edu
}

\section{Background}

This research grant was originally awarded a total of $\$ 814,000$ for a three year project, in FYs 2000-2002. Almost inmediately after receiving funding additional funding years for the program were cut so that in effect the project work was done with the support initially awarded for the first year, i.e., $\$ 262,999$. The work reported here was performed by Profs. Ortega and Lee over the period 1999-2002, and was financed in part by this DOE grant and in part by other sources. In particular, Prof. Ortega's work also received funding from the National Science Foundation and from some industry sources (e.g., Hughes Research Labs and ST Microelectronics.) Due in part to the change in funding level the scope of the project was limited and we focused a significant percentage of the effort on topics related to video transmission. This report summarizes the work accomplished and points to publications that resulted from this work; these publications are in many cases available from our webpages or can be sent upon request.

\section{Scope of proposed work}

Current networks show increasing heterogeneity both in terms of their bandwidths/delays and the applications they are required to support. This is a trend that is likely to intensify in the future, as real-time services, such as video, become more widely available and networking access over wireless links becomes more widespread. For this reason we propose that application-specific proxies, intermediate network 
nodes that broker the interactions between server and client, will become an increasingly important network element. These proxies will allow adaptation to changes in network characteristics without requiring a direct intervention of either server or client. Moreover, it will be possible to locate these proxies strategically at those points where a mismatch occurs between subdomains (for example, a proxy could be placed so as to act as a bridge between a reliable network domain and an unreliable one.) This design philosophy favors scalability in the sense that the basic network infrastructure can remain unchanged while new functionality can be added to proxies, as required by the applications. While proxies can perform numerous "generic" functions, such as caching or security, we concentrate here on media-specific, and in particular video-specific, tasks.

The goal of this project was to demonstrate that application- and network-specific knowledge at a proxy can improve overall performance especially under changing network conditions. We summarize below the work performed to address these issues. Particular effort was spent in studying caching techniques and on video classification to enable DiffServ delivery. Other work included analysis of traffic characteristics, optimized media scheduling, coding techniques based on multiple description coding, and use of proxies to reduce computation costs. This work covered much of what was originally proposed but with a necessarily reduced scope.

\section{Scheduling}

One popular approach to enable media transmission over unreliable networks is to use scalable or multiresolution compression techniques. In our work we have studied scheduling techniques for video encoded using such multiresolution methods. The basic problem we solve is the following: assume video is represented by a number of layers, with each lower layer being needed in order to decode the higher layers; then in which order should packets corresponding to different layers be sent. For example, when considering two consecutive video frames, is it better to send first the base layers of both frames? or is it preferable to transmit all layers of the first frame followed by all layers of the second frame? The answer is that the transmission order depends on the channel conditions. For example, transmission of the base layers first may be preferable when channel conditions are poor.

We have investigated this issue and developed algorithms that enable efficient, yet nearly optimal, decisions on which packets to transmit. These decisions are based on information available about the video data, including both the rate-distortion characteristics of specific data packets, as well as the dependencies between packets. This leads us to estimate the "expected run-time distortion" of the video data and use this information to select packets that will provide the maximum reduction in distortion $[1,2,3]$. It is important to note that our recent work [3] has shown that these algorithms can be very efficiently implemented. Thus for pre-encoded data, even network proxies should be able to handle the computation load required for the proposed channel adaptive scheduling.

\section{Multiple Description Coding}

The basic idea of these techniques is to produce compressed media data where some redundancy has been preserved so that, when transmission occurs over an unreliable channel, decoding can be performed even if losses occur. As an example, if two redundant versions, or descriptions, are transmitted, then it is possible to decode based if either one of the descriptions is received. Note that this in contrast with scalable or multiresolution techniques (such as those discussed in the previous section) where the data is divided into layers and a layer can only be decoded if the lower resolution layers were received.

In particular in this project we explored techniques for rate control in a multiple description setting, which could be easily deployed in network proxies. In our work [4] we demonstrated that the level of redundancy could be targeted to meet specific packet losses characteristics. Thus at either a server or at a network proxy it would be possible to run algorithms that would transmit data with the "right" amount of redundancy. 


\section{Selective Caching}

Caching has been recognized as one of the primary roles to be played by network proxies. In this project we investigated the problem of caching video data and concentrated on a selective caching technique, where only certain video frames are kept in the cache, under the assumption that memory limitations precluded the whole video sequence being stored. We designed two algorithms to prioritize the frames to be stored by considering two different scenarios, one where quality of service guarantees are available, the other where only best effort transmission is available $[5,6]$.

When reliable transmission is available from server to proxy we assume that the goal of the caching algorithm is to minimize the cost of using the reserved bandwidth, under the assumption that the client will have to request reliable bandwidth for whatever portion of the video sequence that is not present at the proxy. In the best effort case we assume that the link between proxy and client is reliable, while that between proxy and server is not. In this situation we assume that the video data is being stored in a decoder buffer and our goal is to design a caching algorithm that will minimize the probability of decoder buffer underflow. Given that it is not possible for the caching algorithm to know a priori what the network conditions will be, our algorithm is based on determining for each sequence the frames at which highest risk of decoder buffer underflow occurs (i.e., those where the decoder buffer is expected to be closest to empty even when channel conditions are good). Our results demonstrate that in both cases a network proxy is able to provide improved performance.

\section{Proxy-based appproaches for client computation reduction}

We have also studied how network proxies can help reduce the computation load at low energy/compute power clients. More specifically we have studied how a proxy can analyze image/video data and provide the client with information to speed up the computation of the inverse discrete cosine transform (DCT) [7]. The trade-off we explore is one where it is possible to increase computation at the proxy and increase slightly the transmitted bit rate, in order to achieve reductions of the computation at the client. Specifically, for the IDCT this entails having several reduced complexity IDCT implementations at the clients, with the proxy analyzing the data to be decoded and informing each client about which IDCT should be chosen for each part of an image.

\section{Integration with DiffServ}

The Internet carries increasingly diverse applications requiring different levels of network quality of service (QoS) including continuous media (CM) applications, which have more stringent quality of service (QoS) requirements than traditional TCP-based applications. Internet QoS remains a challenging task as we face a variety of application requirements, even if the bandwidth will become abundant and cheap.

To provide some level of service quality, the current best-effort Internet must be supplemented with the ability to differentiate traffic from different service classes and to realize different service levels for different service classes. The Differentiated Services framework (e.g., the IETF-Diffserv) intends to provide appropriate grades of service to different applications having different requirements with relatively simple yet scalable methods. The deployment of such service differentiating networks and the end-system applications' clever use of service features will greatly benefit multimedia applications. There has been little research focusing on the QoS interaction between continuous-media (CM) applications and QoS enabled networks. This project explored such positive interactions between the multimedia applications and the service differentiating networks. We present a futuristic QoS mapping framework to combine application-level and network-level effort for QoS support as a result this project.

We present a video categorization scheme based on the concept of relative priority index (RPI), which is made on the basis of the content segments' QoS needs, that effective QoS mapping between categorized application streams and network DiffServ levels can be developed. RPI plays a good bridging tool in 
enabling the network to be content-aware to achieve better end-to-end video quality. Practical guidelines of effective QoS mapping is derived based on the categorized RPI under a total cost constraint. Then, a dynamic QoS mapping control scheme, including feedforward and feedback QoS control, is studied for DiffServ networks. To achieve reliable and consistent end-to-end CM streaming with relative service differentiation, we develop a dynamic and aggregate QoS mapping control scheme with packet, session, and class-based granularity levels for categorized packets at the CM gateway, which is located at the edge of the DiffServ domain. In particular, we focus on dynamic QoS control to handle QoS demand variations of $\mathrm{CM}$ applications (e.g. varying priorities from aggregated/categorized packets) and QoS supply variations of the DiffServ network (e.g. varying loss/delay due to fluctuating network loads). These results were documented in $[8,9]$.

\section{Traffic Analysis and Modeling}

As the traffic behavior is an important factor shaping the environment of the internet and thus affecting QoS planning, we studied the basic nature of self-similarity of traffic exiting from a content provider's server. In addition, we studied reliability issues in planning the content provision through the network. The results were presented in publications [10, 11, 12].

We have also studied mechanisms to enable a media sender, or a proxy, to predict future packet losses based on currently observed packet losses. In this work we demonstrated how techniques adapted from universal source coding could be applied to achieve an efficient prediction tool [13].

\section{Bibliography}

[1] Z. Miao and A. Ortega, "Optimal scheduling for streaming of scalable media," in Proc. of Asilomar Conf. on Signals, Systems and Computers, (Pacific Grove, CA), Oct.-Nov. 2000.

[2] Z. Miao and A. Ortega, "Expected run-time distortion based scheduling for delivery of scalable media," in Proc. of Packet Video Workshop '02, (Pittsburgh, PA), Apr. 2002.

[3] Z. Miao and A. Ortega, "Fast adaptive media scheduling based on expected run-time distortion," in Proc. of Asilomar Conf. on Signals, Systems and Computers, (Pacific Grove, CA), Nov. 2002.

[4] P. Sagetong and A. Ortega, "Optimal bit allocation for channel-adaptive multiple description coding," in Proc. of SPIE Image and Video Communications and Processing, (San Jose, CA), pp. 53-63, Jan 2000.

[5] Z. Miao and A. Ortega, "Scalable proxy caching for streaming video services over QOS and besteffort networks," IEEE J. on Sel. Areas in Comm., Special issue on Internet Proxy Services, vol. 20, pp. 1315-1327, Sept. 2002. Accepted for publication.

[6] Z. Miao and A. Ortega, "Proxy caching for efficient video services over the internet," in Proc. of Packet Video Workshop '99, (New York, NY), Apr. 1999.

[7] W. Pan, A. Ortega, I. Hajj-Ahmad, and R. Sannino, "Proxy-based approaches for idct acceleration," in Proc. of Visual Comm. and Image Processing '01, (San Jose, CA), Jan. 2001.

[8] J. Shin, J. Kim, D. C. Lee, and C.-C. J. Kuo, "Adaptive packet forwarding for relative differentiated service and categorized packet video," in Proc. IEEE ICC' 2001, (Helsinki, Finland), June 2001.

[9] J. Shin, J. Kim, D. C. Lee, and C.-C. J. Kuo, "Dynamic quality of service mapping framework for relative service differentiation-aware media streaming," in Proc. International Conference on Information Technology: Coding and Computing, pp. 30-34, Apr. 2001. 
[10] D. C. Lee, "Edge effect on first interadmission time in optimal queue scheduling," in Proc. Communication Networks and Distributed Systems Modeling and Simulation Conference, (San Diego, CA), Jan. 2000.

[11] D. C. Lee, "Reliability measures for networks with predetermined traffic," in Proc. Communication Networks and Distributed Systems Modeling and Simulation Conference, (San Diego, CA), Jan. 2000.

[12] D. C. Lee, "Self-similarity and queueing behavior of traffic exiting from a server pool," in Proc. Symposium on Performance Evaluation of Computer and Telecommunication Systems, (San Diego, CA), Jul. 2002.

[13] R. Singh and A. Ortega, "Modeling of temporal dependence in packet loss using universal modeling concepts," in Proc. of Packet Video Workshop '02, (Pittsburgh, PA), Apr. 2002. 